\title{
A Review on Peer-to-Peer Live Video Streaming Topology
}

\author{
Ammar Waysi AlTuhafi \\ National Advanced IPv6 Center \\ (NAv6) - Universiti Sains \\ Malaysia
}

\begin{abstract}
Peer-to-peer systems become a promising alternative for traditional server-client system to providing video streaming; the topology is the one of most important part of these systems. This paper talking about the simple concept of topology in $\mathrm{p} 2 \mathrm{p}$ live video streaming and presents a review for $\mathrm{p} 2 \mathrm{p}$ live video streaming topology, in this paper three main types of topology that most used in $p 2 p$ live video streaming systems has been discussed and shows their strength and weakness for each one of them, beside briefing for 18 studies tried to hybrid between two of the main types or using some artificial intelligence tools to improve topology performance.
\end{abstract}

\section{General Terms}

Computer Network, peer-to-peer.

\section{Keywords}

p2p; peer-to-peer; topology; video streaming; video live.

\section{INTRODUCTION}

P2p network is a network built over the physical network to overcome the limitation of server-client systems. Hence, the p2p network uses physical network routing and forwarding functions. In a $\mathrm{p} 2 \mathrm{p}$ network, peers cooperate to provide services to each other; thus, peers are simultaneously clients and servers. P2p networks are usually built in the application layer. Peers in the $\mathrm{p} 2 \mathrm{p}$ network are connected via logical links, and the link between two peers may take several hops in the physical network. These links between peers construct the path of the payload direction in the $\mathrm{p} 2 \mathrm{p}$ network called the p2p network topology [1].

A p2p network is formed by choosing a subset of physical network nodes. The connection between these subset nodes are peer links. The links between peers have different methods and procedures of selection. This has an impact on $\mathrm{p} 2 \mathrm{p}$ network quality and performance. These links comprise the so-called $\mathrm{p} 2 \mathrm{p}$ network topology. P2p network topology can be divided into two main types: unstructured and structured topologies [2].

In unstructured topology peers can join and leave with usually some determinants like sending request to joining and leaving and selecting its neighbors, as an unstructured $\mathrm{p} 2 \mathrm{p}$ network does not require information on the physical network [3]. An unstructured $\mathrm{p} 2 \mathrm{p}$ network is based on a random graph to choose the connection for each peer; the most common topology in unstructured $\mathrm{p} 2 \mathrm{p}$ network is the mesh topology [4]. The structured topology is tightly controlled, and there is no randomization in peers' arrangement. Each peer is organized into a structured graph and each peer registers with serves, providing information that is required by the server, the most famous and useable type of structured topology is tree topology [5]. The most important feature in a structured $\mathrm{p} 2 \mathrm{p}$ network is distributing hash table (DHT), which define the structure of $\mathrm{p} 2 \mathrm{p}$ network. The task also, maintains peers data in this structure, and routes data between peers [6].

\section{MAIN TYPES OF PEER-TO-PEER TOPOLOGY}

There are three main types of peer-to-peer topology, these types are: single tree, multi tree, and mesh topology; almost one or more of these topologies are used in any peer-to-peer system even is there some modification or enhancement on them, to know the principles of these topologies we will discuss each one of them [7].

\subsection{Single-Tree Topology}

Single tree topology is a structured topology wherein peers participating in live video streaming session form a tree structure at the application layer, with the video source server acting as the root of this tree. Every peer in this tree becomes a part of a certain level. In tree topology, each peer receives the video from its parent peer at one level above and forwards the received video to its children peers at one level below; Figure 1 shows the tree topology [8].

Many algorithms may be used for constructing this type of topology, putting every peer in the suitable level, and choosing the parent and children. All these algorithms attempt to decrease the levels of the tree by increasing the number of peers per each level [9]. The reason behind the reduction of the tree levels is to reduce the number of hops taken by the chunks, and thus reduce video delay, especially at lower levels [10].

Although the tree topology is a good structure for video live streaming, it still has two drawbacks. The first is when a peer gets off the video streaming, its children and descendant peers will also be taken off. The server can detect the peer getting off either through sign-off signal or using time-out inference. The second drawback is the occurrence of tree leafs. Leafs contribute only in downloading, and are passive in uploading. Studies have estimated that leaf peers comprise about $30 \%$ of the entire peers in the tree. At the same time, the tree topology is simple to construct, and usually involves only two factors, namely, parent selection and loop detection and avoidance $[11,12]$.

There few techniques have been proposed to solve peer joining and leaving issue; in peer joining there are the following techniques:

- All peers in the network have constant maximum number of children; every joining peer try to connect to peer has children less than the maximum number of children. 
- Using round robin method to add joining peer to the peers in the network; the server applying these method to all peers in the network one by one.

- The joining peer try to connect with peer has the most similar bandwidth.

For leaving peer there is little technique too:

- The grandfather peer will be the responsible for providing children peers of the leaved peer.

- One of the children peers of the leaved peer will take the father peer place and one of its children will be in its old place and so on until the end of the tree.

- All peers of the branch from leaved peer to the end will connect directly to the server[13].

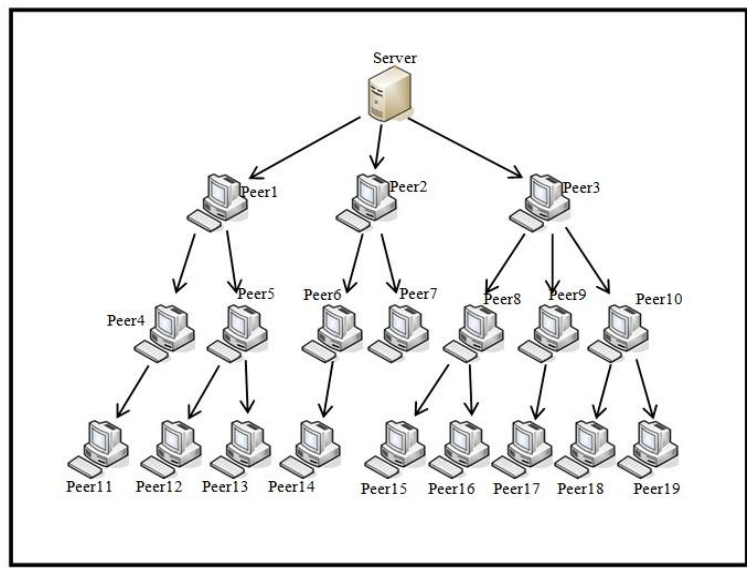

Fig 1: Single-Tree Topology

\subsection{Multi-Tree Topology}

Multi-tree topology is an unstructured topology, in which there are more than one sub-tree instead of one streaming tree. The video streaming is divided by the server to multiple substreams and each sub-stream provides one of the sub-trees. Although we call them sub-trees, each sub-tree has all the peers but in different order and every peer has different positions in different sub-trees. Each sub-stream flows in its own sub-tree form server to leafs. The purpose of multi-tree topology is to fix the passive leafs' problem in single tree topology because the leaf in some sub-tree is middle peer in another sub-tree. Another problem in which a sub-tree is solved is when peer becomes off, because the children peers can receive video streaming from another sub-tree. Figure 2 shows Multi-tree topology [14].

The number of levels of Multi-tree topology like single tree topology many studies tries to reduce the levels of each tree to the minimum [15]. We can consider multi-tree topology as a combination between the simplicity of tree topology and unstructured topologies. This topology has two drawbacks: The first is increasing the overhead of the streaming compared to tree topology. The second occurs when a peer becomes a leaf in all sub-trees and contributes only in downloading without uploading [16]; to solve last drawback, an algorithm has been suggested for joining peers in multi tree become middle peer in only one sub-tree and leaf for other sub-trees [17].

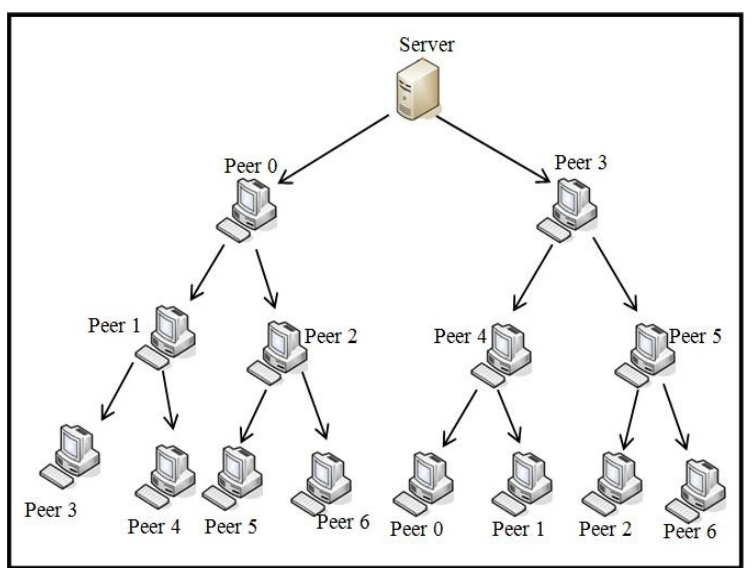

Fig 1: Multi-Tree Topology

\subsection{Mesh Topology}

Mesh topology is one of the unstructured topologies where peers can join and leave dynamically by establishing connection with the neighbors and disconnecting it at any given time. In mesh topology, peers download video streaming from multiple neighbors' peers and upload video streaming to multiple neighbors at the same time. If one of the peer's neighbors leaves and stops the connection with the peer, the peer can still download and upload video streaming from/to other neighbors. Mesh topology has high flexibility against the peers who have sequences of on and off state, or what we call the churn problem [18].

Although most peers-to-peers systems which using mesh topology based on random choosing for the neighborhoods and represent it in random graph, other systems tries to make some determinants in selecting peers in these neighborhoods and to do connection between each pair peers according to these determinants or agreement [19].

Different topologies comprise different policies of the connection such as how many peers to make a connection and which peers should they connect to, etc. The peering decisions are usually based on the peer's functions and resource availability on both peers, such as the number of connections of peers, bandwidth, CPU and memory usage. Peers in mesh topology not only make a connection as a reaction to neighbor peers leaves, but also change neighbors optionally to reach better performance. Figure 3 shows mesh topology [20].

Choosing better neighbors for each peer in mesh topology improve the video chunk exchange between neighbors. The decision of choosing neighbor relationship is mostly based on the following:

- The available resources in the neighborhood peers, such as the number of peers connected with the two peers upload and download bandwidth, CPU and memory usage, etc.

- The link quality between every two peers which can be characterized by transmission delay and packet loss rate.

- The video parts are complementary, which means that each peer in the neighborhood has video chunks needs than other neighbor peers and vice versa [21]. 


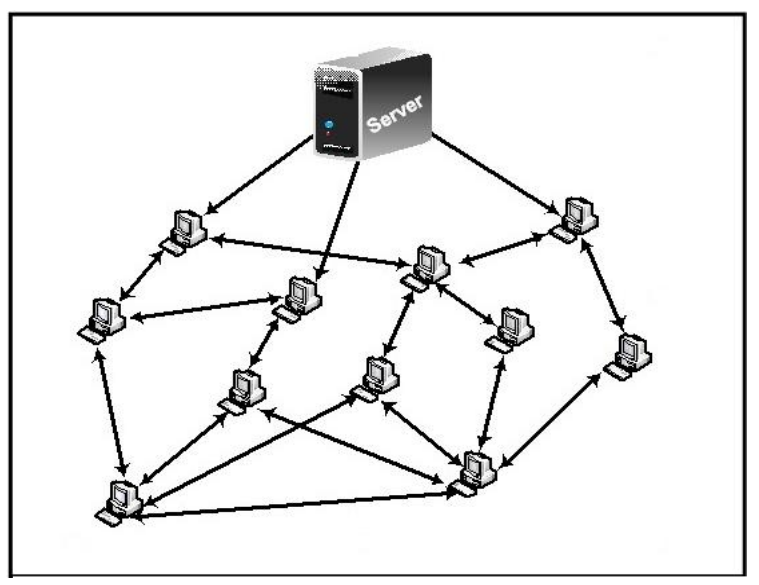

Fig 3: Mesh Topology

\section{DISTRIBUTED HASH TABLE (DHT)}

The DHT is the main difference between structured and unstructured topologies in the p2p system [5], which has been invented to eliminate flooding messages in large-scale filesharing p2p systems such as Chord [22], Tapestry [23], Pastry [24], and CAN [25]. These flooding messages consume high bandwidth and processing of networks [4]. The main service of DHT is the lookup operation, a hash function search used in the lookup operation. This operation is based on the value associated with any given key. The hash function reduces searching time [26].

The DHT is used to manage the distribution of data among peers in structured $\mathrm{p} 2 \mathrm{p}$ systems. The DHT also saves an updating list of the current peers by adding and removing the peers' IDs from the list, which is based on the joining and living of peers with their resources and assigning a suitable task for each peer [6]. The use of DHT in live video $p 2 p$ systems has become popular because of the advantage of the use of the list of peers and its resources in broadcasting videos as efficiently as possible [27].

\section{TOPOLOGIES MODIFICATIONS}

Numerous studies have attempted to apply several modifications on the three main topologies to obtain a more efficient topology or to cover the drawbacks of one of these topologies by applying a particular method, algorithm, or a hybrid between two topologies. These studies are shown below.

\subsection{Topology Optimized Algorithm}

In this study, a new structured algorithm was proposed to construct a p2p live video-streaming topology. The algorithm is an optimization algorithm based on the minimummaximum k-means clustering. The algorithm takes information used in the clustering from the peers' communication history, and then groups the peers in the network to the k-clusters with each cluster in the network having its own center peer. The algorithm used in the minimum-maximum principle enhances the performance of the chunks' hops. Each peer in every cluster is connected with the center peer in its own cluster.

The figure below shows the diagram of this topology. A super peer can record all the subnet center nodes, which function as a server of other cluster center nodes. The performance of this topology is tested with 3,000 peers. The complexity of routing hops is $\mathrm{O}(1)$, and the update routing table time is $\mathrm{O}(\mathrm{N} 2)$ [28].

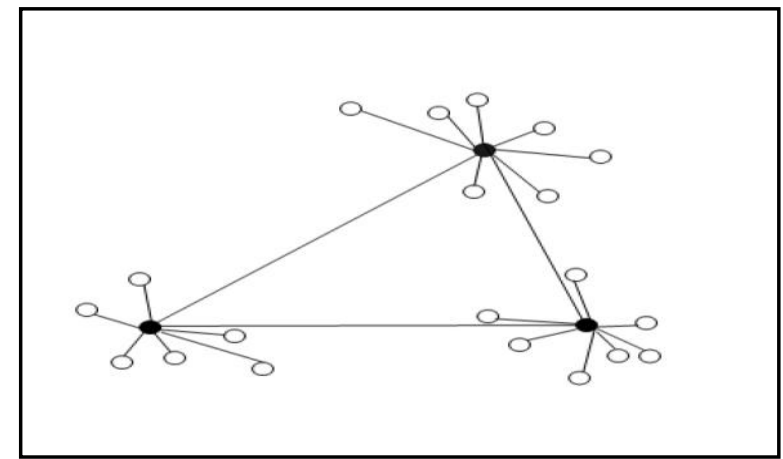

Fig 4: K-Mean Topology

\subsection{Hybrid Model based on Genetic Algorithm Topology (HCGA)}

This study attempts to solve the tree's level optimization in video-streaming through a new hybrid model by utilizing two well-known artificial intelligence tools, namely, k-means clustering and genetic algorithm. Two steps are required to obtain the solution. First, the model is represented as a multiobjective optimization model. Second, the clustering problem is modeled using a genetic algorithm to obtain better results for the clustering operation.

The results of the experiments were obtained by applying different configurations of the tree topology, which resulted in a better performance of data routing. The experiments were conducted through a simulation of 200 nodes, and the result was compared with the topology based on only the k-mean clustering [9].

\subsection{Adaptive Overlay Topology}

In this study, a simple and fully distributed mechanism was proposed to construct and maintain a $\mathrm{p} 2 \mathrm{p}$ live video-streaming topology based on the mesh topology. The main contribution of the topology is the automatic movement of the peers, which have a large bandwidth, close to the video source server based on the measured chunk-delivery performance of the peers rather than on the theoretical bandwidth specifications, this study does not shows the method of this operation. The contribution not only reduces the chunk delivery delay with peers having a high bandwidth but also propagates the improvement all over the network.

The results of this study were obtained through a simulation. The results show a performance improvement of up to $50 \%$ compared with that of the regular mesh topology. The proposed topology also has a robust performance against peerchurning and network problems because the method used in this study is distributed and operates continuously [29].

\subsection{Minimum Delay Mesh Topology (MDM)}

This study focuses on the optimization of the overlay construction for $\mathrm{p} 2 \mathrm{p}$ streaming systems. The study also maximizes the total downloading rate of the heterogeneous peers' download and uploads in the tree topology for live $\mathrm{p} 2 \mathrm{p}$ streaming systems. The problem is formalized into a graph problem that can determine the maximum number of edgedisjointed trees and centralize the heuristic algorithm applied to solve the problem. The result shows the following benefits: 
- The use of the uplink bandwidth of each single peer with their heterogeneous downloads is made efficient through the receipt of the live video in different bitrates.

- The distributed algorithm is proposed to construct an adaptive $p 2 p$ topology for live video-streaming by minimizing the video delay in peers. The delay is reduced by $30 \%$, and link stress is lessened [30].

\subsection{Optimal Topology Design for Overlay Networks:}

The topology used in this study depends on two main factors. The first factor is formatting the problem to establish new overlay links by using the Integer Linear Programming (ILP). The second factor is proposing heuristics to choose a nearoptimal overlay topology.

ILP depends on the mathematical linear equation, whereas proposing heuristics depends on three heuristic methods as follows:

- Greedy Heuristic: This heuristic depends on the choice of a sequence of nodes. The first node chooses the node that has the best neighbor to minimize the cost of establishing an overlay link.

- Node Clustering Heuristic: This heuristic depends on grouping the node in a decentralized way. Every group has a leader node, which has the highest degree, a relay node, which is the node with a connection with two or more leader points, and the remaining nodes called the ordinary nodes [31].

\subsection{Topology Optimization}

This study is a proposed hybrid topology that combines tree and mesh topologies. The peers chosen to belong to the tree topology are stable peers in the network, which are called treebone in this study. The unstable peers overcome the high effectuation of peer-leaving on the tree topology instead of using all the available bandwidths of the network. The time that a peer spends in the network without going off, which is called the age threshold and can be calculated based on a mathematical question, was studied to determine if a peer is stable or unstable.

The simulation results of this study were compared with the regular mesh and tree topologies. The results show a significant improvement, and were verified through real implementation in the PlantLab organization [32].

\subsection{Smart-Fit}

This study tries to minimize the overall number of video chunk hops in the $\mathrm{p} 2 \mathrm{p}$ network. The hops represent the video receiving delay on the peers. This study attempts to place each joining peer in a suitable place in the structured tree based on two factors:

- The uplink capacity of each single peer, and

- The predication of peer behavior.

The proposed topology attempts to predicate each peer behavior by determining the previous behavior of the peer over different periods of time. The topology was then reconstructed based on the predication result. The simulation result of this study shows a $19 \%$ improvement in the video delay and a $10 \%$ improvement in packet-losing in poor network conditions compared with the traditional tree topology [34].

\subsection{Adaptive Overlay Topology Optimization (AOTO)}

The Adaptive Overlay Topology Optimization (AOTO) technique attempts to modify the tree topology by constructing a tree between each video source peer and its neighbors. In doing so, harmony between the physical network and the $\mathrm{p} 2 \mathrm{p}$ network may be achieved by choosing the closest peer in the physical network as a neighbor in the $\mathrm{p} 2 \mathrm{p}$ network.

This topology does not require the global information of all the $\mathrm{p} 2 \mathrm{p}$ networks when each peer in the network attempts to organize the peers in its neighborhood. The simulation shows that the AOTO topology has $55 \%$ better performance in reducing traffic compared with the regular tree peer-to-peer topology [35].

\subsection{Mesh-Based Over Tree-Shape (MBOTS)}

In this study, a modification of the tree topology was proposed, where a mesh topology was added to the tree structure topology. Each level of the tree contained from the nodes had the same distance, which is the number of video chunk hobs from the video server to the peers, from the video server. The peers in each level of the tree topology were connected by a mesh topology connection, which reduces the load of the peers' resources by providing multiple parents to each peer in the network.

The simulation results show better performance than the standard tree topology. Adding a mesh topology to the tree structure solves the issue of the tree's leaf peers by exchanging a video chunk among the peers, which allows video chunk to upload and download [36].

\subsection{Maximum Average Bandwidth Spanning Tree (MABST)}

A tree topology, which has been modified to a special type called the Maximum Average Bandwidth Spanning Tree (MABST), was used in this study. The study attempts to optimize the average of the system stream rate through two steps:

- MABST is proven to be a NP-complete problem through reduction from the Hamiltonian Path problem.

- A special and efficient heuristic algorithm derived from the greedy algorithm was then proposed.

The results were obtained from both real implementation and simulation. The real implementation was conducted by utilizing 20 computers (peers), whereas the simulation was conducted by utilizing different sizes of the network node. The performance was improved compared with the naïve greedy algorithm [33].

\subsection{Hybrid Overlay Approach with Tree Optimization (HOWTO)}

This study is concerned about the hybrid between the mesh and tree topologies. The peers were divided by groups in the mesh topology part. Each group contained peers with the same ISP (internet service provider) and bandwidth. The server obtains this information by joining message of each peer. The tree part topology was constructed over the mesh part by promoting the peers from the mesh topology. The peers in the mesh topology are promoted if the peers exhibit a stable behavior for a long period and have high bandwidths. These peers are moved nearer to the video server because the 
tree topology has the same depth in its branches. Figure 5 shows the HOWTO topology.

The result of this study was obtained through simulation. These results were not compared with other topologies. Only the time delay of joining peers and the chunk delivery delay were estimated with a set of 100 routers and 1000 nodes [37].

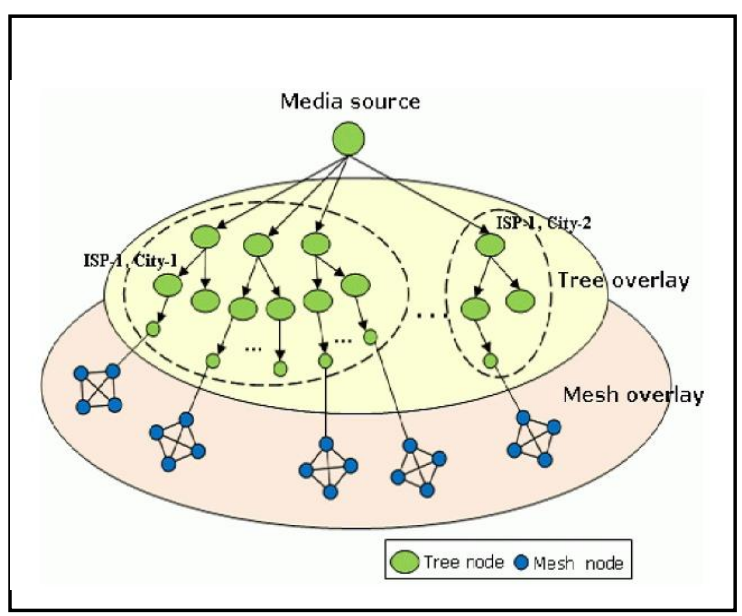

Fig 5: HOWTO Topology

\subsection{Ring Tree (HRT)}

Ring topology is used in $\mathrm{p} 2 \mathrm{p}$ file sharing systems. This type of topology is structured. The peers are organized as a ring with each peer passing the information to the next until the data reaches a particular peer [38].

This study combines the tree and ring topologies. The aim of the study is to solve the peer joining and leaving issue in the tree topology, which allow a quick recovery from peer leaving as well as smooth delivery and reduced delay time of the video. The solution was achieved by adding backup links, which are links between a father peer and the children peers belonging to the father peer's brother. The children peers can receive a video from another father peer if the father peer leaves. The other father peer provides for its children peers as well as the children peers of the original father peer.

The peers that belong to the same father have a ring topology connection among them. This type of connection aids in receiving a video from more than one peer. The experimental results were applied on 100 peers. These results were not compared with other topologies. However, the times of delay, network bootstrapping, and the peers joining and leaving are shown [39]. Figure 6 shows the HRT topology.

\subsection{Layered Ring Topology (LRing)}

In this study, a layered ring topology was proposed. Each layer is contained in a single ring topology. The aim of this study is to dispose of the disadvantages of the tree and mesh topologies such as the peer churn issue in the tree topology and the network overhead issue in the mesh topology. The proposed topology is structured, easily implemented, and can easily maintain the peer churn issue. The other advantage of the layered ring topology is the ability to balance the video load in the network efficiently.

A redundant model was also proposed in this study to increase the collaboration between clients for a better performance. The simulation result for the 250 nodes compared with the single ring topology shows that the layered ring topology performs $18 \%$ better than the single ring topology [40].

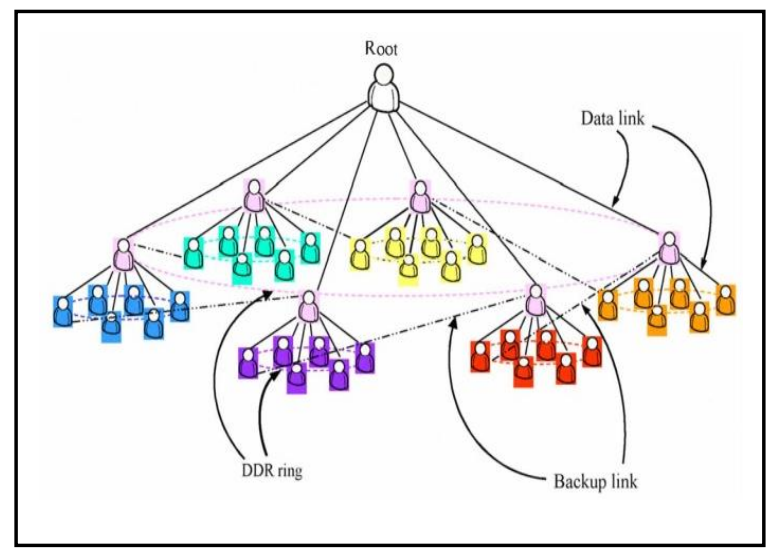

Fig 6: HRT Topology

\subsection{Layered Ring Topology (LRing)}

In this study, a layered ring topology was proposed. Each layer is contained in a single ring topology. The aim of this study is to dispose of the disadvantages of the tree and mesh topologies such as the peer churn issue in the tree topology and the network overhead issue in the mesh topology. The proposed topology is structured, easily implemented, and can easily maintain the peer churn issue. The other advantage of the layered ring topology is the ability to balance the video load in the network efficiently.

A redundant model was also proposed in this study to increase the collaboration between clients for a better performance. The simulation result for the 250 nodes compared with the single ring topology shows that the layered ring topology performs $18 \%$ better than the single ring topology [40].

\subsection{Adaptive Topology Formation}

In this study, two techniques were proposed to construct an adaptive topology. The first is based on the source of video streaming. The second is based on topology adaptation. An adaptive $\mathrm{p} 2 \mathrm{p}$ streaming video playback rate was proposed. The video rate changes depending on the result of the local network measurements to obtain better video quality compared with the bandwidth of the peers.

The adaptive topology is based on the Markov chain Monte Carlo method by increasing the connection for each peer until the outcome video is balanced with the upload bandwidth of the peers. Each peer has neighbors that are suitable to the peer's ability. The simulation results were not compared with other topologies, but a mathematical model was proposed and compared with the simulated results to prove the results [41].

\subsection{ZIGZAG Tree Topology}

The ZIGZAG technique was invented to improve tree topology performance. This type of tree topology is based on 
the divided peers in each level to the clusters with an equal number of peers in each cluster. Each cluster has a head peer belonging to a higher level. This head peer does not participate in the video distribution in this cluster. Instead, the head peer controls and manages the cluster as well as distributes the video chunk to another cluster in the next level. The ZIGZAG separates video distribution and peer management. This technique reduces the effect of the leaving peers, which retains the effects in the local area [42]. Figure 7 shows the ZIGZAG technique.

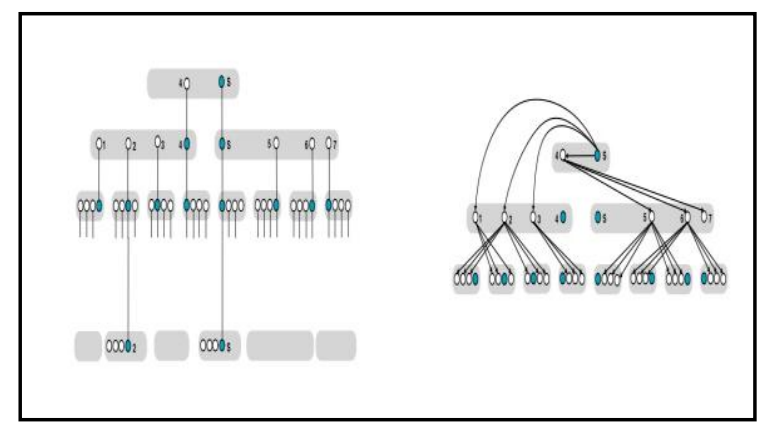

Fig 7: ZIGZAG Topology

\subsection{MeshTree Topology}

In this study, a tree topology is constructed as the backbone for the entire topology. A mesh topology is then built over the tree topology to reduce tree level and increase robustness against peer joining and leaving issues.

A new joining peer can be added as part of the backbone tree, delivery tree, or pure mesh. The delivery tree is the intermediate topology between the backbone tree and pure mesh. To improve the performance of the topology, each peer tries to add a new link with other peers. This operation happens periodically. If the new link achieves better performance without affecting other links between two peers, then the new link will be accepted; otherwise, the link will be dropped. The simulation result exhibits better performance than a regular tree topology [43].

\subsection{An Adaptable Topology for Live Video Streaming}

In this study, a hybrid topology was proposed between the tree and mesh topologies. A fast peer with an upload bandwidth of more than double that of the video bitrate becomes a part of the tree topology; otherwise, the peer becomes a part of the mesh topology.

The study proposes a method to determine the maximum children peers ability of each peer in the tree topology part by adding one peer each time and determining if the time exceeds $\mathrm{T}$ time. The $\mathrm{T}$ time is equal to one second if the size of the video chunk is equal to the video bitrate. The initial results show that the proposed topology appears to be a tree topology when the number of peers with high bandwidths increases and when the video bitrate is small. The proposed topology acts like a mesh toplogy when peers with high bandwidths decrease and when the video bitrate is large. Figure 8 shows the adaptable topology for live video-streaming [44].

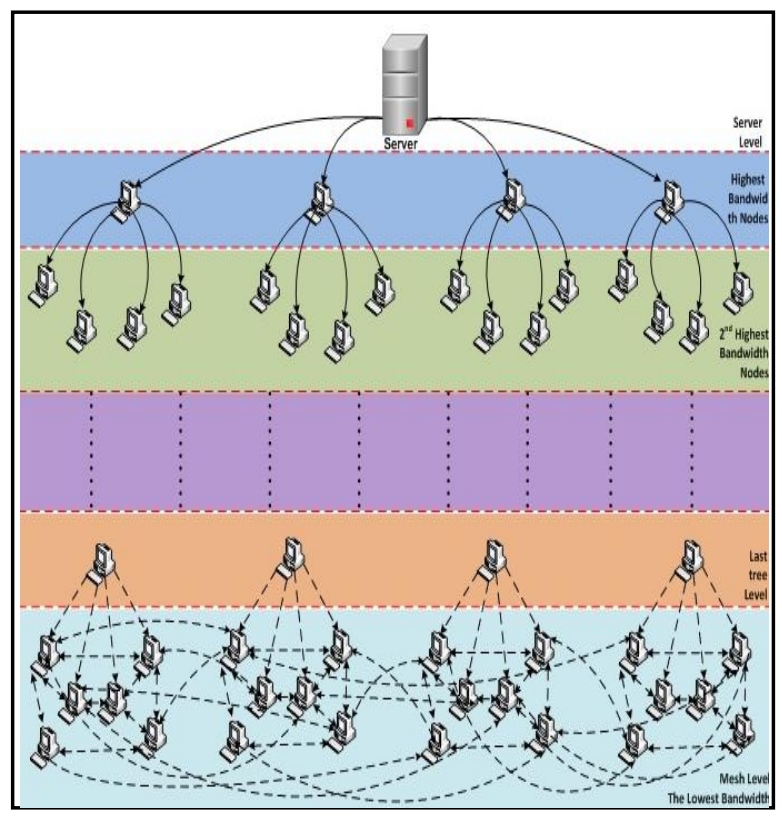

Fig 8: Adaptable Topology

\section{Conclusion}

The topology of $\mathrm{p} 2 \mathrm{p}$ live video streaming involves virtual lines that connect peers over the application layer to transfer video data, with each peer acting as a server-client in the $p 2 p$ network. The following conclusions are drawn from the review results. The tree topology is efficient in packet distribution, whereas the mesh topology incurs very long delays. The tree topology is sensitive to link and peer failure, whereas the mesh topology has a reliable behavior with dynamic alteration. The tree topology is usually designed for a large group with one source, whereas the mesh topology is usually designed for a large group with one or more sources. The properties and performance of the multi-tree topology lie between the tree and mesh topology. Tree and multi tree topology used push-up mechanism for data delivery, while mesh topology used pull-down mechanism. Finally, the multitree topology has close performance to directed mesh topology. Table I shows the summary of the three main types of the peer-to-peer topology for live video streaming.

Studies that deal with modified topologies that discussed in this paper are based on these three topologies with some modification, these modifications done by using some of artificial intelligence algorithms, mathematical tools, or hybrid between two of the main topologies. Table II shoes the summary of these studies. 
Table 1. The summary of main types of peer-to-peer live video streaming topologies

\begin{tabular}{|c|c|c|c|c|c|c|c|}
\hline $\begin{array}{c}\text { Topology } \\
\text { Type }\end{array}$ & Delay & Peers Types & $\begin{array}{l}\text { Peer Failure } \\
\text { or peer leave }\end{array}$ & $\begin{array}{c}\text { Network } \\
\text { Overhead }\end{array}$ & $\begin{array}{c}\text { Servers } \\
\text { Number } \\
\end{array}$ & $\begin{array}{c}\text { Data } \\
\text { Delivery }\end{array}$ & Implementation \\
\hline Tree & $\begin{array}{l}\text { Low } \\
\text { delay }\end{array}$ & $\begin{array}{l}\text { Has good } \\
\text { performance with } \\
\text { peers have high } \\
\text { bandwidth, but has } \\
\text { not good performance } \\
\text { with peers have low } \\
\text { bandwidth. }\end{array}$ & $\begin{array}{l}\text { Has week } \\
\text { robustness } \\
\text { and resilience } \\
\text { against peer } \\
\text { leaving or } \\
\text { peer failure. }\end{array}$ & $\begin{array}{l}\text { Low } \\
\text { network } \\
\text { overhead. }\end{array}$ & $\begin{array}{l}\text { Usually } \\
\text { used with } \\
\text { one server. }\end{array}$ & $\begin{array}{l}\text { Used push- } \\
\text { down data } \\
\text { delivery. }\end{array}$ & $\begin{array}{l}\text { Implementing } \\
\text { tree easier than } \\
\text { multi-tree and } \\
\text { mesh. }\end{array}$ \\
\hline Multi-tree & $\begin{array}{c}\text { Medium } \\
\text { delay }\end{array}$ & $\begin{array}{l}\text { Has good } \\
\text { performance with } \\
\text { peers have high } \\
\text { bandwidth, with } \\
\text { better performance } \\
\text { with peers have low } \\
\text { bandwidth than tree } \\
\text { topology. }\end{array}$ & $\begin{array}{l}\text { Has medium } \\
\text { robustness } \\
\text { and resilience } \\
\text { against peer } \\
\text { leaving or } \\
\text { peer failure. }\end{array}$ & $\begin{array}{l}\text { More than } \\
\text { tree and } \\
\text { less than } \\
\text { mesh. }\end{array}$ & $\begin{array}{l}\text { Used for } \\
\text { one server } \\
\text { or more. }\end{array}$ & $\begin{array}{l}\text { Used push- } \\
\text { down data } \\
\text { delivery for } \\
\text { each sub- } \\
\text { tree. }\end{array}$ & $\begin{array}{l}\text { Implementing } \\
\text { multi-tree more } \\
\text { complicated than } \\
\text { tree and easier } \\
\text { than mesh. }\end{array}$ \\
\hline Mesh & $\begin{array}{l}\text { High } \\
\text { delay }\end{array}$ & $\begin{array}{l}\text { Has good } \\
\text { performance with } \\
\text { peers have high, low, } \\
\text { or even } \\
\text { heterogeneous } \\
\text { bandwidth. }\end{array}$ & $\begin{array}{l}\text { Has high } \\
\text { robustness } \\
\text { and resilience } \\
\text { against peer } \\
\text { leaving or } \\
\text { peer failure. }\end{array}$ & $\begin{array}{l}\text { High } \\
\text { network } \\
\text { overhead. }\end{array}$ & $\begin{array}{l}\text { Used for } \\
\text { one server } \\
\text { or more. }\end{array}$ & $\begin{array}{l}\text { Used pull- } \\
\text { up data } \\
\text { delivery. }\end{array}$ & $\begin{array}{l}\text { Implementing } \\
\text { mesh more } \\
\text { complicated than } \\
\text { multi-tree and } \\
\text { tree. }\end{array}$ \\
\hline
\end{tabular}

Table 2. The summary of main types of peer-to-peer live video streaming topologies

\begin{tabular}{|c|c|c|}
\hline Topology Name & Topology Based on & Additional Techniques \\
\hline Optimized Algorithm & Mesh & Minimum-maximum k-means clustering. \\
\hline HCGA & Tree & K-means clustering and genetic algorithm. \\
\hline Adaptive Overlay & Mesh & Changing position of peers according to their performance \\
\hline MDM & Mesh & Maximum number of edge-disjointed in graph. \\
\hline Optimal Topology & Mesh & ILP and heuristics for choose a near-optimal overlay topology. \\
\hline Topology Optimization & Tree and Mesh & $\begin{array}{l}\text { Age threshold to evaluate peer to become a part of tree or mesh } \\
\text { topology. }\end{array}$ \\
\hline MABST & Tree & NP-complete and Greedy algorithm. \\
\hline Smart-Fit & Tree & Place each joining peer in a suitable place. \\
\hline АОТО & Tree & $\begin{array}{l}\text { Constructing a tree between each video source peer and its } \\
\text { neighbors and choosing the closest peer in the physical network as } \\
\text { a neighbor in the p } 2 \mathrm{p} \text { network. }\end{array}$ \\
\hline MBOTS & Tree and Mesh & $\begin{array}{l}\text { Peers in each level of tree connected with each other as a mesh, } \\
\text { and have same distance from video server. }\end{array}$ \\
\hline HOWTO & Tree and Mesh & $\begin{array}{l}\text { Clustering peers in the same ISP and same bandwidth in groups } \\
\text { connecting each other. }\end{array}$ \\
\hline HRT & Tree and Ring & $\begin{array}{l}\text { A peer connects to father peer of its brother peer beside its } \\
\text { original father. }\end{array}$ \\
\hline LRing & Ring & Dividing peers to multi layer rings. \\
\hline Adaptive Topology & Mesh & $\begin{array}{l}\text { Video rate changes depending on peers bandwidth and Markov } \\
\text { chain Monte Carlo method. }\end{array}$ \\
\hline ZIGZAG & Tree & $\begin{array}{l}\text { Divided peers in each level to the clusters with an equal number of } \\
\text { peers in each cluster and each cluster have a head peer belonging } \\
\text { to a higher level. }\end{array}$ \\
\hline MeshTree & Tree and Mesh & $\begin{array}{l}\text { Monitoring the performance of peer when adding new peer to its } \\
\text { neighbors. }\end{array}$ \\
\hline
\end{tabular}




\section{REFERENCES}

[1] L. Yunhao, et al., "Location-aware topology matching in P2P systems," in INFOCOM 2004. Twenty-third AnnualJoint Conference of the IEEE Computer and Communications Societies, 2004, pp. 2220-2230 vol.4.

[2] Z. Li and P. Mohapaira, "The impact of topology on overlay routing service," in INFOCOM 2004. Twentythird AnnualJoint Conference of the IEEE Computer and Communications Societies, 2004, p. 418.

[3] P. Hyojin, et al., "A Survey on Peer-to-Peer Overlay Network Schemes," in Advanced Communication Technology, 2008. ICACT 2008. 10th International Conference on, 2008, pp. 986-988.

[4] D. Doval and D. O'Mahony, "Overlay networks: A scalable alternative for P2P," Internet Computing, IEEE, vol. 7, pp. 79-82, 2003.

[5] M. Waldvogel and R. Rinaldi, "Efficient topology-aware overlay network," ACM SIGCOMM Computer Communication Review, vol. 33, pp. 101-106, 2003.

[6] A. T. Gai and L. Viennot, "Broose: a practical distributed hashtable based on the de-Bruijn topology," in Peer-toPeer Computing, 2004. Proceedings. Proceedings. Fourth International Conference on Peer-to-Peer Computing, pp. 167-174, 2004, pp. 167-174.

[7] G. Yue, et al., "Survey on Scheduling Technologies of P2P Media Streaming," Journal of Networks, vol. 6, pp. 1129-1136, 2011.

[8] B. Hudzia, et al., "Treep: A tree based p2p network architecture," in In Cluster Computing, 2005. IEEE International (pp. 1-15). IEEE., 2005, pp. 1-15.

[9] Z. LianQing and L. Jun, "A Hybrid Clustering Model for Hierarchical Overlay Topology," in Natural Computation, 2009. ICNC'09., 2009, pp. 111-114.

[10] M. Amad, et al., "GPM: A generic and scalable P2P model that optimizes tree depth for multicast communications," International Journal of Communication Systems, 2008.

[11] Y. Chu, et al., "A case for end system multicast," Selected Areas in Communications, IEEE Journal on Selected Areas in Communication (JSAC), vol. 20, pp. 1456-1471, 2002.

[12] J. Jannotti, et al., "Overcast: reliable multicasting with on overlay network," in Proceedings of the 4th conference on Symposium on Operating System Design \& Implementation-Volume 4, 2000, pp. 14-14.

[13] H. Deshpande, et al., "Streaming live media over a peerto-peer network," Technical Report, 2001.

[14] V. Venkataraman, et al., "Chunkyspread: Multi-tree Unstructured Peer-to-Peer Multicast," IPTPS 2006 Program, 2006.

[15] C. Liang, et al., "Topology optimization in multi-tree based P2P streaming system," in Tools with Artificial Intelligence, 2009. ICTAI'09. 21st International Conference on tools with artificial intelligence, pp.806813., 2009, pp. 806-813.

[16] M. Castro, et al., "SplitStream: high-bandwidth multicast in cooperative environments," in In ACM SIGOPS
Operating Systems Review (Vol. 37, No. 5, pp. 298-313). ACM., 2003, pp. 298-313.

[17] W. Tu, "Performance Analysis for Overlay Multicast on Tree and MD Mesh Topologies," in Communications, 2007. ICC'07. IEEE International Conference on, 2007, pp. $6433-6438$.

[18] Y. Liu, et al., "A survey on peer-to-peer video streaming systems," Peer-to-peer Networking and Applications, vol. 1, pp. 18-28, 2008.

[19] T. Fuhrmann, "On the topology of overlay-networks," in Networks, 2003. ICON2003. The 11th IEEE International Conference on Networks (ICON), pp. 2712762003 , pp. 271-276.

[20] J. Ghoshal, et al., "Network architectures for live peer-topeer media streaming," Technical report, Department of Computer Science and Engineering, University of Nebraska-Lincoln, 2007.

[21] J. Liu, et al., "Opportunities and challenges of peer-topeer internet video broadcast," Proceedings of the IEEE, vol. 96, pp. 11-24, 2008.

[22] I. Stoica, et al., "Chord: A scalable peer-to-peer lookup service for internet applications," ACM SIGCOMM Computer Communication Review, vol. 31, pp. 149-160, 2001.

[23] B. Y. Zhao, et al., "Tapestry: An infrastructure for faulttolerant wide-area location and routing," 2001.

[24] A. Rowstron and P. Druschel, "Pastry: Scalable, decentralized object location, and routing for large-scale peer-to-peer systems," In Middleware 2001 (pp. 329350). Springer Berlin/Heidelberg, 2001, pp. 329-350.

[25] S. Ratnasamy, et al., "A scalable content-addressable network," ACM SIGCOMM Computer Communication Review, vol. 31, pp. 161-172, 2001

[26] A. Ghodsi, "Distributed k-ary system: Algorithms for distributed hash tables," PhD These, KTH-Royal Institute of Technology, 2006.

[27] J. Li, et al., "Implementing aggregation and broadcast over Distributed Hash Tables," ACM SIGCOMM Computer Communication Review, vol. 35, pp. 81-92, 2005.

[28] Y. Ma, et al., "A P2P network topology optimized algorithm based on minimum maximum k-means principle," in Hybrid Intelligent Systems, 2009. HIS'09., 2009, pp. 396-399.

[29] R. J. Lobb, et al., "Adaptive overlay topology for meshbased p2p-tv systems," in Proceedings of the 18th international workshop on Network and operating systems support for digital audio and video, 2009, pp. $31-36$

[30] M. Yang and Y. Yang, "Optimal overlay construction on heterogeneous live peer-to-peer streaming systems," in Parallel Processing (ICPP), 2010, pp. 690-698.

[31] M. Youssef, et al., "Optimal topology design for overlay networks," IFIP Networking 2007, 2007.

[32] W. Feng, et al., "mTreebone: A Hybrid Tree/Mesh Overlay for Application-Layer Live Video Multicast," in Distributed Computing Systems, 2007. ICDCS '07. 27th 
International Conference on Distributing Computing Systems 2007, pp. 49-49.

[33] R. Li, et al., "On topology construction in layered P2P live streaming networks," in NOMS2010, 2010, pp. 599606.

[34] P.-J. Wu, "Overcoming Packet Loss in Peer-to-Peer Video Streaming Systems," PhD These, National Sun Yat-Sen University, 2009.

[35] L. Yunhao, et al., "AOTO: adaptive overlay topology optimization in unstructured P2P systems," in Global Telecommunications Conference, 2003. GLOBECOM '03. IEEE, 2003, pp. 4186-4190 vol.7.

[36] S. Awiphan, et al., "Mesh-based data delivery over multiple tree-shaped routes in P2P overlay network," in Information Networking, 2009. ICOIN 2009., 2009, pp. $1-5$.

[37] H. Byun and M. Lee, "HOWTO: a hybrid overlay approach with tree optimization," in Computer Science and Information Engineering, 2009 WRI World Congress on Computer Science and Information Engineering, pp. 311-315, 2009, pp. 311-315.

[38] A. Shaker and D. S. Reeves, "Self-stabilizing structured ring topology p2p systems," in In Proceedings of the Fifth IEEE International Conference on Peer-toPeer Computing (P2P 2005), pp. 39-46, 2005, pp. 39-46.

[39] H. Nen-Fu, et al., "Construction of an efficient ring-treebased Peer-to-Peer streaming platform," in Networked
Computing and Advanced Information Management (NCM), 2010 Sixth International Conference on, 2010, pp. $75-80$.

[40] W. Jigang, et al., "LRing: A Layered Ring Topology for Reliable Streaming," in 1st International Workshop on Ad-hoc Ambient Computing, 2008.

[41] H. Luan, et al., "Adaptive topology formation for peerto-peer video streaming," Peer-to-peer Networking and Applications, vol. 3, pp. 186-207, 2010.

[42] D. A. Tran, et al., "Zigzag: An efficient peer-to-peer scheme for media streaming," in In INFOCOM 2003 Twenty-Second Annual Joint Conference of the IEEE Computer and Communications. IEEE Societies (Vol. 2, pp. 1283-1292). IEEE., 2003, pp. 1283-1292.

[43] S. W. Tan, et al., "MeshTree: Reliable low delay degreebounded multicast overlays," in Parallel and Distributed Systems, 2005. Proceedings. 11th International Conference on Parallel and Distributed Systems Workshops (ICPADS'05)- Volume 02, 2005: pp. 565-569. , 2005, pp. 565-569.

[44] A. W. AlTuhafi, et al., "Framework for Adaptable Topology for Peer-to-peer Live Video Streaming," Accepted to be published in IEEE Business, Engineering and Industrial Applications Colloquium 2013 (IEEE BEIAC 2013), 2013. 